\title{
Twistable Dipolar Aryl Rings as Electric Field Actuated Conformational Molecular Switches
}

\author{
Kílian Jutglar Lozano ${ }^{1}$, Raul Santiago ${ }^{1}$, Jordi Ribas-Arino ${ }^{1 *}$, Stefan T. Bromley ${ }^{1,2^{*}}$ \\ ${ }^{1}$ Departament de Ciència de Materials i Química Física \& Institut de Química Teòrica i \\ Computatcional (IQTCUB), Universitat de Barcelona, c/ Martí i Franquès 1-11, 08028 \\ Barcelona, Spain
}

${ }^{2}$ Institució Catalana de Recerca i Estudis Avançats (ICREA), Passeig Lluis Companys 23, 08010

Barcelona, Spain

*Corresponding authors: j.ribas@ub.edu, s.bromley@ub.edu

The ability to control the chemical conformation of a system via external stimuli is a promsing route for developing molecular switches. For eventual deployment as viable sub-nanoscale components that are compatible with current electronic device technology, conformational switching should controllable by a local electric field (i.e. E-field gateable) and accompanied by a rapid change in conduction. In organic chemical systems the degree of $\pi$-conjugation is linked to the degree of electronic delocalisation, and thus largely determines the conductivity. Here, by means of accurate first principles calculations, we study the prototypical biphenyl based molecular system in which the dihedral angle between the two rings determines the degree of conjugation. In order to make this a gateable system we create a net dipole by asymmetrically functionalising one ring with electronegative substituents $(\mathrm{F}, \mathrm{Br}$ and $\mathrm{CN})$ with different polarisabilities. In this way, the application of an E-field interacts with the dipolar system to influence the dihedral angle, thus controlling the conjugation. For all three considered substituents we consider a range of E-fields, and in each case extract conformational energy profiles. Using this data we obtain the minimum E-field required to induce a barrierless switching event for each system. We further extract the estimated switching speeds, the conformational probabliities at finite temperatures, and the effect of applied E-field on electronic structure. Consideration of these data allow us to assess which factors are most important in the design of efficient gateable electrical molecular switches. 


\section{Introduction}

Use of external stimuli (e.g. light, temperature, $\mathrm{pH}$ ) to controllably induce molecular level structural and/or chemical changes can be exploited for using individual molecules like switches in electronic devices. ${ }^{1,2,3}$ Unlike the top-down fabrication of inorganic silicon-based transistors, molecular switches are synthesised via bottom-up chemical design. As such, there are many different examples of switchable molecular systems based on a wide range of chemistries. ${ }^{4}$ Since electric field (E-field) driven semiconductor-based switches are key components in electrical circuitry, in recent years extensive research efforts have been devoted to fabricating junctions of single-molecules or self-assembled monolayers whose conductance can be reversibly switched by means of external E-fields. Large and reversible jumps of conductance triggered by E-fields can be achieved by means of changes in the redox state of electrochemically active molecules ${ }^{5,6,7}$, molecular conformational rearrangements ${ }^{8,9,10,11,12,13}$, and structural changes in the electrode-molecule contacts. ${ }^{14,15}$ Electrically controlled switching is also possible through E-field-induced molecular tautomerizations, isomerizations, conformational changes or rotations in molecular crystals ${ }^{16}$, in solution ${ }^{17}$, in frozen molecular solids ${ }^{18}$, in molecules adsorbed on surfaces ${ }^{19,20,21,22,23,24}$ and in encapsulated molecules. ${ }^{25}$ It is noted that external E-fields can even catalyze bond rupture processes ${ }^{26}$ or more complex reactions at the single-molecule level ${ }^{27,28,29}$.

Although molecular switches are an attractive route towards extreme miniaturization, to eventually compete as viable transistors they must be: i) highly responsive to modest E-fields, and ii) able to control the transport of charge carriers. Herein, we focus in one of the most promising molecular building blocks for single-molecule electronics, namely, biphenyl (see Fig. 1a). In an elegant single-molecule junction study, a set of different substituted biphenyl molecules with variable degrees of steric hindrance between the two aryl rings allowed for the measurement of the transport properties of the biphenyl moiety as a function of the relative twist angle between the two rings. ${ }^{30} \mathrm{It}$ was found that conformational planarity favours through-bond $\pi$-conjugation and higher conductance, whereas relatively twisted conformations are highly resistive (see Fig. 1b). ${ }^{30,31}$ More recent experiments have shown that thermal fluctuations of the torsion angle of phenyl groups in junctions containing bipenyl moieties result in stochastic conductance switching ${ }^{32}$ while an E-fieldinduced variation of the torsion angle results in a controlled conductance switching. ${ }^{11,13}$ Specific values of the torsion angle together with appropriate electron-donating and withdrawing substituent groups in biphenyl-containing junctions have been predicted to lead to molecular rectifiers. ${ }^{33}$ Besides the single-molecule electronics context, the torsion angle between aryl rings in biphenyl moieties is also a key factor to bear in mind for optimal performance of organic functional materials..$^{34,35,36,37}$ In fact, 
changes of this torsion angle in response to external stimuli is a powerful tool to develop switchable materials. ${ }^{38,39}$

Here, we asymmetrically functionalise one of the two rings of biphenyl (i.e. X in Fig. 1) to make a dipolar system that intrinsically is conformationally responsive to applied E-fields. We note that twistable dipolar aryl rings have been proposed as a possible basis for E-field actuated molecular switching in hypothetical 2D graphene-graphyne hybrids. ${ }^{39}$ Other modelling studies have shown that mono-substituted benzene rings also hold potential for E-field actuated molecular rectifiers ${ }^{40}$ or switches $^{41}$ at the single-molecule level. Herein, we use accurate computational modelling to study how to optimise twistable dipolar aryl rings for deployment as molecular switches by comparing three different dipolar functionalisations $(\mathrm{X}=\mathrm{F}, \mathrm{Br}, \mathrm{CN})$. Specifically, we consider the energetic contributions due to molecular conformational and permanent/induced dipoles for a range of twist angles $(\theta)$ and applied E-field strengths $(\mathbf{F})$. We also consider switching speed and the effectiveness of the switching at a range of temperatures. Thereby, we provide an overview of which factors are most important for designing low field E-field actuated molecular conformational switches at finite temperatures.

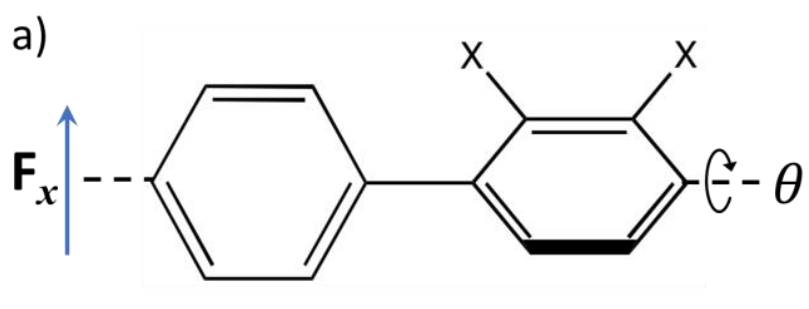

b)

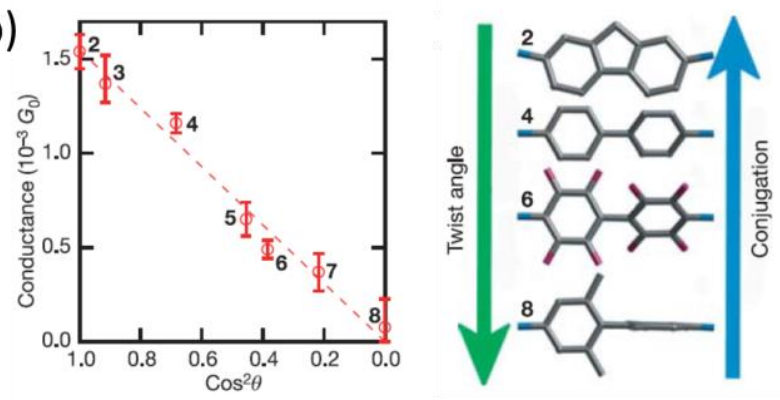

Figure 1. a) General chemical structure of the model biphenyl systems used herein, where $\mathbf{F}_{\boldsymbol{x}}$ is the applied E-field vector in the $x$-direction, $\theta$ is the conformational twist angle, and the $\mathrm{X}$ are the functional dipole-inducing substituents $(X=F, B r, C N)$. The left ring is orientationally fixed in the $x-y$ plane and the right ring is geometrically unconstrained. $b$ ) Relationship between conformational twist angle $(\theta)$ and conductance in biphenyl ring systems (figure adapted with permission from ref 30 ),

\section{Methodology}

The energies and dipoles of each functionalized bi-phenyl system (i.e. with $X=F, B r, C N$ were computed using density functional theory (DFT) based calculations employing the hybrid PBEO functional and a $6-31+\mathrm{G}(\mathrm{d})$ basis set. ${ }^{42,43}$ using the Gaussian 09 code. ${ }^{44}$ In each case we consider 
conformations resulting from varying $\theta$ from $0^{\circ}$ to $180^{\circ}$ in steps of $5^{\circ}$, where the angle of the nonfunctionalised aryl ring is kept fixed at $0^{\circ}$. Constrained optimisations for each conformation were performed with zero applied field, and an E-field applied in the $x$ direction $\left(\mathbf{F}_{\boldsymbol{x}}\right)$ which is taken to be parallel to the fixed aryl ring and perpendicular to the inter-ring bond (see Fig. $1 \mathrm{~b}$ ). We consider $\mathbf{F}_{\boldsymbol{x}}$ values from $0.001-0.015$ a.u. (i.e. $5.1 \times 10^{6}-7.7 \times 10^{7} \mathrm{~V} / \mathrm{cm}$ ) in steps of $0.002 \mathrm{a} . \mathrm{u}$, and a relatively strong field of 0.03 a.u $\left(1.5 \times 10^{8} \mathrm{~V} / \mathrm{cm}\right)$. In the solid state, high powered silicon-based metal oxide semiconductor field effect transistors (MOSFETs), also typically operate using E-field strengths towards the lower bound of this range $\left(\sim 1 \times 10^{6} \mathrm{~V} / \mathrm{cm}^{45}\right)$. However, in (bio)molecular systems intrinsic local E-fields arising from ionic charge distributions (e.g. solvents and enzyme active sites) have typical strengths in the range $1 \times 10^{6}-1 \times 10^{8} \mathrm{~V} / \mathrm{cm} \cdot{ }^{46,47,48}$ Such fields have been used in specifically tailored experiments to induce conformational changes of molecules in solution or deposited on surfaces requiring specific set-ups to generate the E-field (e.g. interfacially trapped ions ${ }^{18}$ or proximity of a STM tip ${ }^{20}$.

Generally, the total energy $\left(E_{\text {tot }}\right)$ of a molecule in an E-field is given by:

$$
E_{t o t}=E^{0}-\sum_{n=1}^{\infty}-\frac{\boldsymbol{F}^{n}}{n !} \cdot \boldsymbol{a}_{\boldsymbol{n}}=E^{0}-\boldsymbol{\mu} \cdot \boldsymbol{F}-\frac{1}{2} \boldsymbol{\alpha} \cdot \boldsymbol{F}^{2}-\frac{1}{6} \boldsymbol{\beta} \cdot \boldsymbol{F}^{3}-\cdots
$$

where $E^{0}$ the energy without E-field, $\boldsymbol{F}$ is the E-field, and $\boldsymbol{\mu}, \boldsymbol{\alpha}, \boldsymbol{\beta}$ are the dipole moment, polarizability and the first hyperpolarizability, respectively. ${ }^{49,50}$ We note that in the case of a weakly polarizable dipolar molecule in a weak E-field, $E_{\text {tot }}$ can be well approximately by only the first two terms of the expansion. Assuming this dipolar approximation, the torque $(\tau)$ on the dipole with respect to an applied E-field is given by:

$$
\tau=\frac{d E_{t o t}}{d \theta}
$$

When the moment of inertia $(I)$ is constant, we also have:

$$
\frac{\tau}{I}=\ddot{\theta}
$$

where $\ddot{\theta}$ is the angular acceleration. We extract $\tau$ from our calculated $\theta$-dependent total energies (eq. 2 ) and solve the differential equation (eq. 3 ) to obtain $\theta$ as a function of time, i.e. $\theta(t)$. Thereby, we provide order of magnitude estimates of switching speeds. See S1 for more details.

The probability of finding different twist angle conformations, $P_{\theta}$, at finite temperatures with different applied fields is also estimated using standard Boltzmann statistical mechanics using these DFT-calculated total energies, i.e. 


$$
P_{\theta}=\frac{e^{\frac{-E_{\theta}}{k_{B} T}}}{Z}, \text { with } Z=\sum_{\theta=0}^{360} e^{\frac{-E_{\theta}}{k_{B} T}}
$$

where the $E_{\theta}$ are total system energies for each considered value of $\theta, k_{B}$ is the Boltzmann constant, $T$ is the temperature, and $Z$ is the partition function.

\section{Results and Discussion}

Considering first the variation of $E_{\text {tot }}$ with respect to $\theta$ for zero applied field, the trends can largely be understood through the interplay between $\pi$-conjugation and steric hindrance. The di-fluoro system shows two similar energy minima for $\theta=45^{\circ}, 135^{\circ}$, separated by a barrier of $6.7 \mathrm{~kJ} / \mathrm{mol}$ at $90^{\circ}$ and higher energy maxima at $\theta=0^{\circ}, 180^{\circ}$ of $>10 \mathrm{~kJ} / \mathrm{mol}$ (see Fig. 2a). For the latter maxima when the two aryl rings are aligned in the same plane, although here $\pi$-conjugation is maximized, the steric hindrance between the close $\mathrm{F}$ and $\mathrm{H}$ substituents is also maximized and this strong repulsive interaction dominates the total energy. Twisting away from these maxima rapidly reduces the steric hindrance and allows for the energy lowering effect of $\pi$-conjugation to stabilize the system. However, $\pi$-conjugation is most favored when the two rings are most aligned and as the rings become more mutually twisted the $\pi$-conjugation decreases. This scenario explains the appearance of the small maximum for a perpendicular ring alignment (i.e. $\theta=90^{\circ}$ ) where $\pi$-conjugation is zero. It is noted that the conformational energy profile obtained for the difluoro system at zero field is in line with the results reported in Ref. 51 for 2-fluorobiphenyl. This general scenario also leads to a very similar zero field total energy curve for the di-nitrile system (see Fig. 2e), where the barrier at $\theta=90^{\circ}$ is found to be $7.1 \mathrm{~kJ} / \mathrm{mol}$. For the di-bromo system the same competition between $\pi$-conjugation and steric hindrance also explains the zero-field curve. However, here the relatively larger $\mathrm{Br}$ atoms cause the steric hindrance to dominate for all $\theta$, resulting in a single minimum at $\theta=90^{\circ}$, where this effect is minimized (see Fig. 2d). In all cases, at zero field an ensemble of any of these three biphenyl systems will exhibit a symmetric distribution of conformational angles with respect to $\theta=90^{\circ}$.

The effect of applying an E-field in a direction parallel to the plane of the fixed ring (i.e. $\mathbf{F}_{\mathbf{x}}$ ) on the conformational energy profiles of these bi-aryl ring systems is shown in detail for the case of the di-fluoro system in Figs. 2a-c. The first clear effect of the applied E-field is to induce an energetic stabilisation of only one minimum. In this way the E-field thus switches the system from a relatively broad ensemble of conformational possibilities to a specific narrower range of conformations. With, with increasing E-field strength the scale of the total energy variation with respect to $\theta$ rapidly dominates the variation of the system energy at zero field. The applied E-field clearly energetically favors conformations with lower values of $\theta$ where the permanent dipole of the F-functionalised ring 
is aligned with the field. However, for higher values of $\theta$ where the permanent dipole is anti-aligned with the field, the total energy is less affected. In fact, apart from the highest field strength considered, the total energy of the anti-aligned di-fluoro system remains close to that of the zero-field case. This behavior can be understood by consideration of the energetic contributions of the permanent dipole (i.e. first term in equation 1 ) and the induced dipole (i.e. second and higher order terms in equation 1) for the di-fluoro system in Figs. $2 b$ and $2 c$ respectively. Note that these data are also provided for the di-bromo and di-nitrile systems in S2.

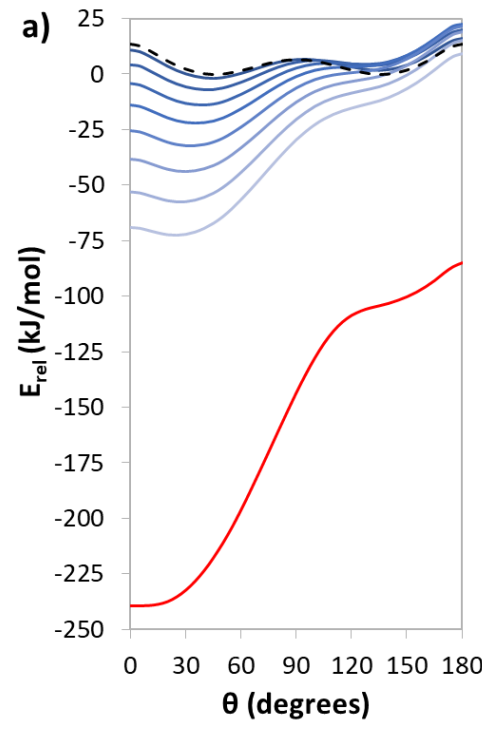

d) 40

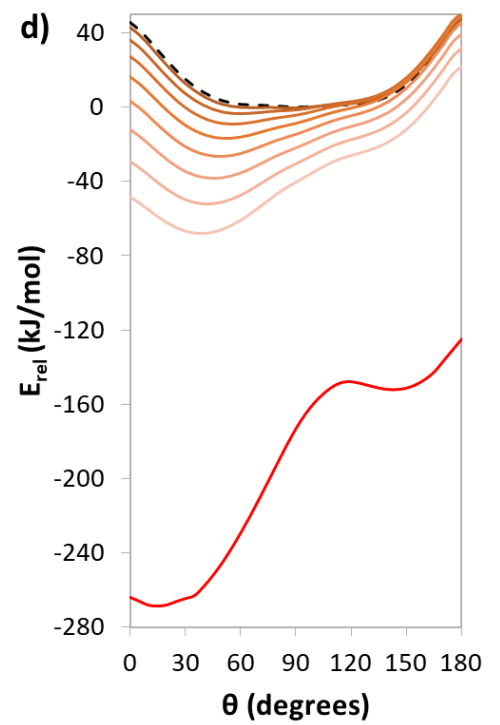

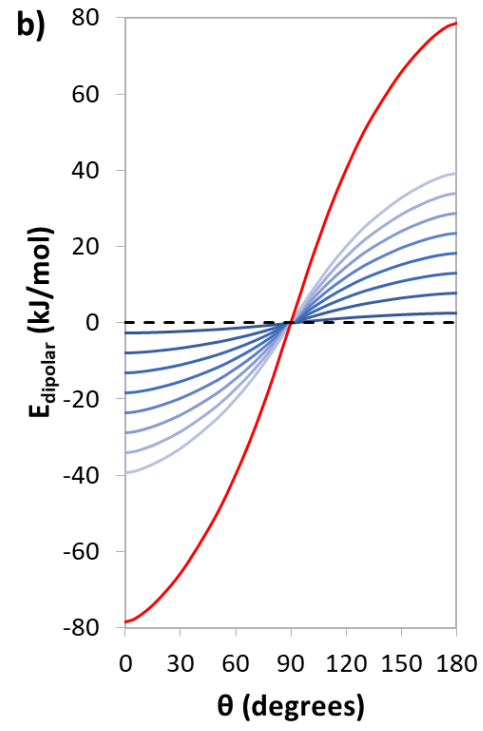
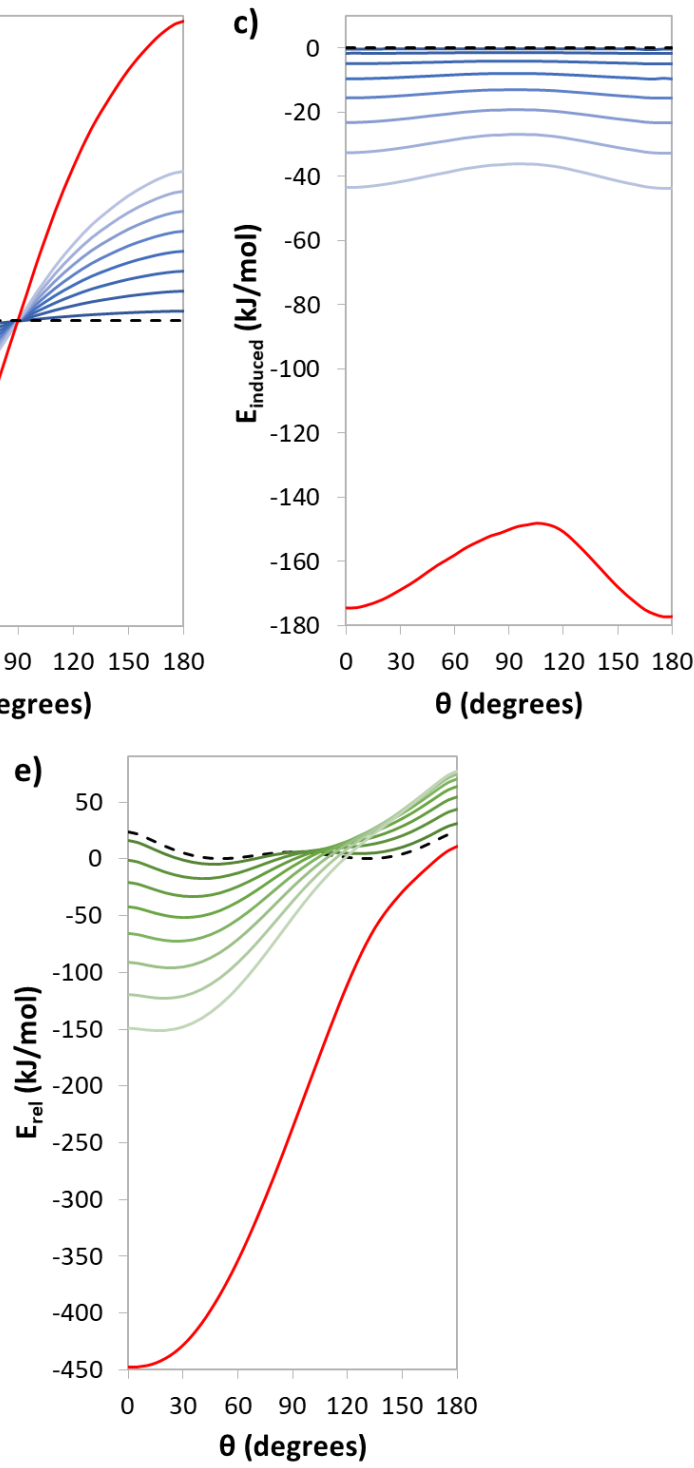

Figure 2. a) Relative total energies $\left(E_{\text {rel }}\right)$ with respect to $\theta$ with relative to the lowest energy zero field (dashed line) conformation of the di-fluoro system. b) Energy contribution due to the permanent dipole (second term in equation 1) for the di-fluoro system. c) Energy contribution due to the induced dipole (third and higher order terms in equation 1 ) for the di-fluoro system. d) $E_{\text {rel }}$ with respect to $\theta$ 
relative to the lowest energy zero field (dashed line) conformation of the di-bromo system. e) $E_{\text {rel }}$ with respect to $\theta$ relative to the lowest energy zero field (dashed line) conformation of the di-nitrile system. In all plots the applied E-field strength varies from $5.1 \times 10^{6}-7.7 \times 10^{7} \mathrm{~V} / \mathrm{cm}$ (dark to light line colour) and the red lines denotes the strongest field of $1.5 \times 10^{8} \mathrm{~V} / \mathrm{cm}$.

The energy due to the permanent dipole is an asymmetric function of $\theta$ with respect to $\theta=$ 90 (i.e. $-\boldsymbol{\mu} . \boldsymbol{F}=-\mu F \cos (\theta)$ ) which equally and oppositely affects the energy for dipolar alignment and anti-alignment, respectively. In Fig. 2c we can see that the contribution from the induced dipolar and higher order terms to the energy is found to vary much less with respect to $\theta$, and is always negative (i.e. energy lowering). For higher field strengths there is a tendency for a more significant energetic effect of the induced dipole for conformations close to full alignment or full anti-alignment of the two aryl rings. For lower field strengths the magnitude of the energetic contribution of the permanent dipole is higher than that of the induced dipole. In this regime induced dipole leads to a slight energetic downshifting of the $\theta$-dependent $\mathrm{E}_{\mathrm{rel}}$ curves but the shapes of the curves are largely unchanged (see S3). However, for field strengths $\geq 6.7 \times 10^{7} \mathrm{~V} / \mathrm{cm}$ the energy lowering from the induced dipole is greater in magnitude than the maximum energy variation due to the permanent dipole. In such cases, the assumption that the field induced energy variation of the system only depends on the orientation of the permanent dipole, will significantly underestimate the effect of the applied E-field. This is in line with the acknowledged importance of the induced dipole in tuning the catalytic effect of external fields in reactivity. ${ }^{28,52}$ For the highest strength E-field considered of $1.5 \times 10^{8} \mathrm{~V} / \mathrm{cm}$, the energy lowering from the induced dipole is more than double the maximum energy variation due to the permanent dipole. Overall, in all systems the energy lowering effect of the induced dipole acts to: i) increase the energy lowering of the permanent dipole when the molecular dipole is aligned with $\mathbf{F}_{\mathbf{x}}$, and ii) largely cancel the energetically destabilising effect of the permanent dipole (or even reverse it at high field) when the molecular dipole is anti-aligned with $\mathbf{F}_{\mathbf{x}}$. In the SI we provide plots of the energetic contributions due to the permanent dipole and the induced dipole interacting with the considered range of E-fields for the di-bromo and di-nitrile systems. 
a)

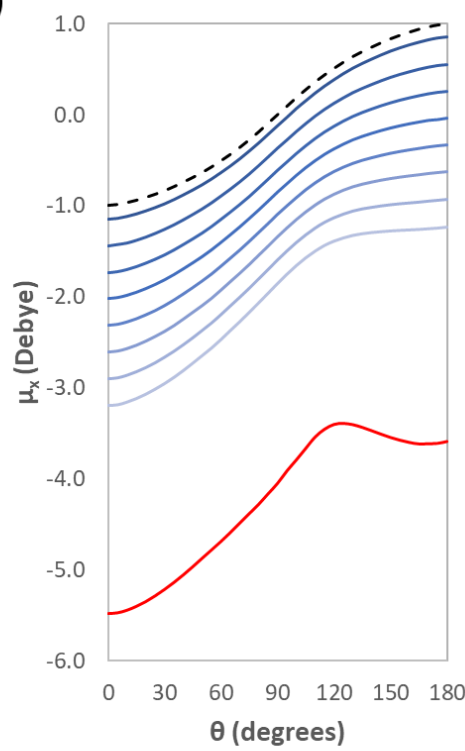

c)

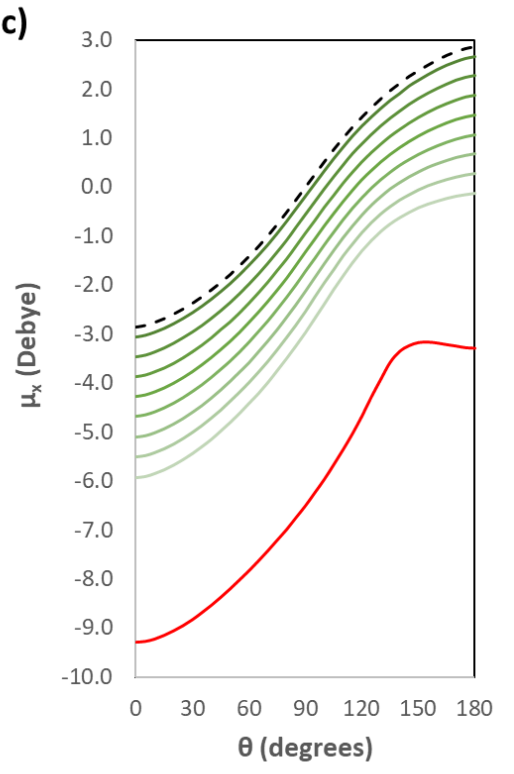

b)

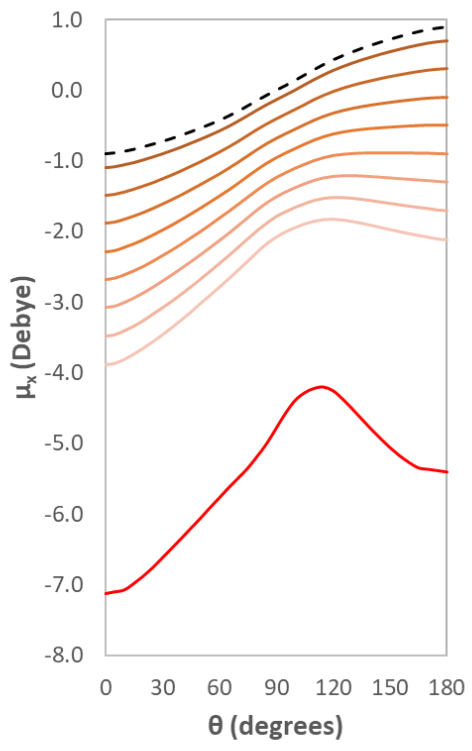

d)

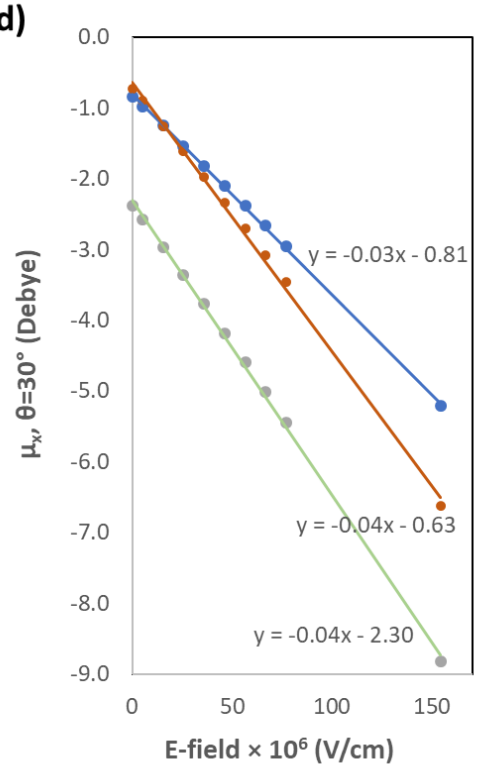

Figure 3. Variation in the $x$-component of the total dipole moment $\left(\mu_{\mathrm{x}}\right)$ with respect to $\mathbf{F}_{\mathbf{x}}$ and $\theta$ for: $\left.\mathrm{a}\right)$ the di-fluoro system, b) the di-bromo-system, and c) the di-nitrile system. In d) we show linear fits to the variation of total dipole moment at $\theta=30^{\circ}$ with respect to increasing E-field strength for each system.

The effect of an applied E-field can also be assessed by examining how it affects the overall the dipole moment. In Figure 3a-c we show how the $x$-component of the total dipole moment varies with respect to increasing field strength and $\theta$ for each system. In terms of total dipole moment the di-fluoro and di-bromo systems show very similar behaviour at zero field and the lowest field strengths. However, with increasing field strength the total dipole in the di-bromo system quickly becomes more negative than the corresponding dipole for the di-fluoro system. This is mainly due to 
the higher polarisability $(\alpha)$ of the larger $\mathrm{Br}$ atoms as compared to the more compact less polarisable F substituents. This difference can be more clearly seen in Fig. $3 \mathrm{~d}$ where the increase in the magnitude of $x$-component of the total dipole moment for each of the three considered systems is plotted with increasing magnitudes of $\mathbf{F}$ for a fixed angle of $\theta=30^{\circ}$. To first order the total dipole can be approximately expressed as the sum of the permanent dipole $\left(\mu_{0}\right)$ and the product between $\alpha$ and applied E-field (i.e. $\boldsymbol{\mu}=\boldsymbol{\mu}_{\mathbf{0}}+\boldsymbol{\alpha F}$ ). The gradient of a linear fit to the data in Fig. $3 \mathrm{~d}$ thus provides us with an estimate of $\alpha$ for each system. Here, we see that although the di-fluoro and di-bromo systems have a similar zero field permanent dipole (i.e. a similar intercept) the steeper linear fit for the latter shows that this system has a significantly higher polarisability. Performing the same analysis for the di-nitrile system shows that the permanent dipole is significantly higher in magnitude than for the dihalogen based systems. Also, the polarisability of the di-nitrile system is almost as high as for the dibromo system. This combination results in this system having the largest magnitude total dipole for all applied field strengths in Fig. $3 \mathrm{~d}$.

The above considerations can help inform us as to which type of system would form the basis for a responsive and efficient molecular switch. Firstly, for each system we identify the minimum applied field which induces a single energy minimum in the $E_{\text {tot }}$ versus $\theta$ curve. We note that although the di-bromo system has a single energy minimum at zero applied field, a small field of $1.5 \times 10^{7} \mathrm{~V} / \mathrm{cm}$ induces a weak double well energy profile which then disappears at slightly higher field strengths. For the di-nitrile system $1.5 \times 10^{7} \mathrm{~V} / \mathrm{cm}$ is already enough to induce a single energy well, whereas a larger field strength of $3.6 \times 10^{7} \mathrm{~V} / \mathrm{cm}$ is required for the di-fluoro system (see Fig. 4). The reason for the dinitrile system being more efficient with respect to this measure (i.e. requires the smallest E-field) is most likely due to the higher permanent dipole in this system. We also note that the E-fields needed to induce a conformational change in our dipolar biphenyl systems are all smaller than those required (ca. $8.0 \times 10^{7} \mathrm{~V} / \mathrm{cm}$ ) to rotate the central phenyl ring of terphenyl derivative without a permanent dipole moment. ${ }^{13}$ This highlights the importance of having large permament dipole moments when it comes to inducing conformational changes by means of modest E-fields.

Using the angular dependence of $E_{\text {tot }}$ at a modest $E$-field of $3.6 \times 10^{7} \mathrm{~V} / \mathrm{cm}$ we can also estimate the order of magnitude of speed at which these systems could function as switches. At this E-field strength the majority ( $66 \%$ ) of the field-induced drop in the total energy is determined by the $\boldsymbol{\mu}$. $\boldsymbol{F}$ term in equation 1 (i.e. the dipolar approximation is reasonable). Solving equation 3 for the interval between the approximate angle of minimum energy $\left(\theta_{0}\right)$ and $\left(\theta_{0}+25\right.$ degrees) we obtain times of 0.21 ps, 0.22 ps, and 0.31 ps for the di-fluoro, di-bromo and di-nitrile systems respectively. 
Although there is some variation in these times all are within a similar narrow range of $0.26 \pm$ $0.05 \mathrm{ps}$ for this $25^{\circ}$ rotation. Assuming this is a rotation speed in these systems we can estimate that a switching cycle from a zero field minimum (taking the $\sim 135^{\circ}$ case for the di-fluoro and di-nitrile systems) to the E-field induced minimum $\left(\sim 35^{\circ}\right)$, and back again, would take approximately $2 \mathrm{ps}$. We note that the fastest commercial CPUs operate with clock speeds (an indication of operations per second) well below $10 \mathrm{GHz} .{ }^{53}$ Considering that in a fast $5 \mathrm{GHz}$ CPU the time period for a full switching cycle is 200 ps, our results indicate that E-field gated molecular conformational change has the potential to provide ultrafast switching speeds.
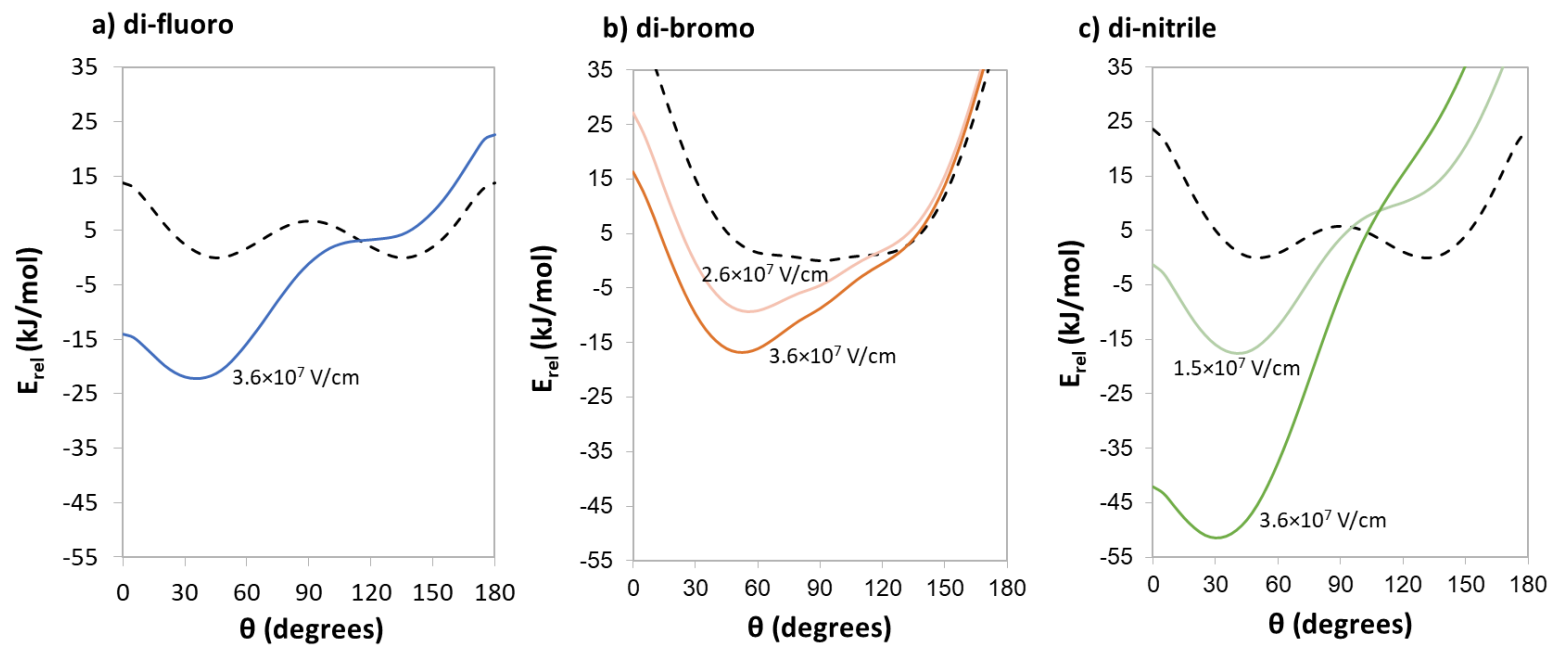

Figure 4. $E_{\text {rel }}$ versus $\theta$ relation for low field strengths for the: a) di-fluoro, b) di-bromo, and c) di-nitrile systems. The lowest field strength shown in each plot denotes the minimum field required to induce a single energy minimum. In all cases the dashed line denotes the zero-field case. For comparison the $E_{\text {rel }}$ behaviour for a field strength of $3.6 \times 10^{7} \mathrm{~V} / \mathrm{cm}$ is also shown in each case 

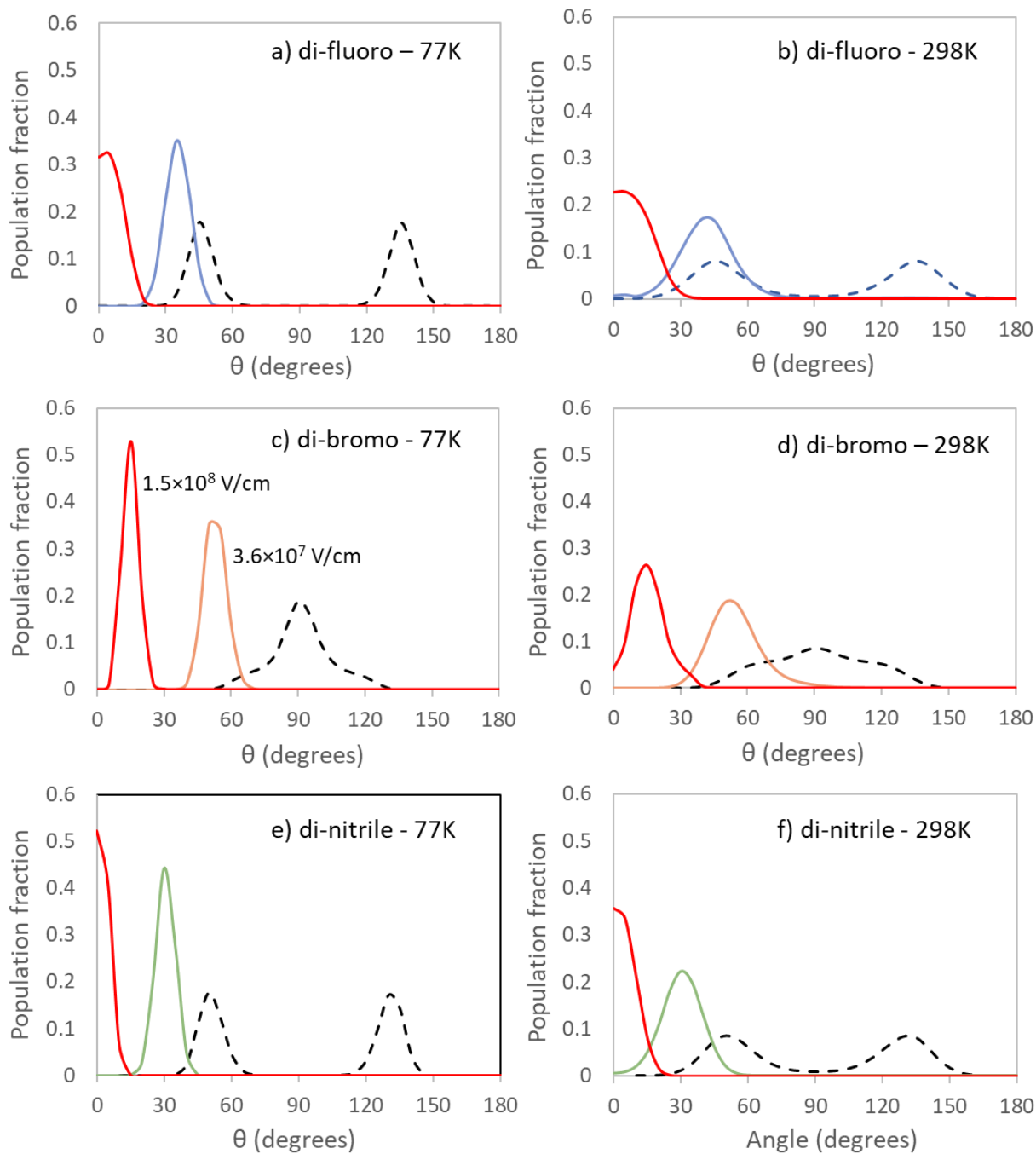

Figure 5. Boltzmann populations with respect to $\theta$ for: a) the di-fluoro system at $77 \mathrm{~K}$ and b) at $298 \mathrm{~K}$, c) the di-bromo system at $77 \mathrm{~K}$, and d) at $298 \mathrm{~K}$, and e) the di-nitrile system at $77 \mathrm{~K}$, and f) at $298 \mathrm{~K}$. For each plot zero field is shown by a dashed line, the high field of $1.5 \times 10^{8} \mathrm{~V} / \mathrm{cm}$ is indicated by a red line and a low field of $3.6 \times 10^{7} \mathrm{~V} / \mathrm{cm}$ is shown by the remaining coloured line.

More pertinent for actual devices is the stabililty of a particular conformational state induced by an applied field at finite temperatures. Here, for two temperatures $(T=77 \mathrm{~K}$ and $\mathrm{T}=298 \mathrm{~K})$ we compute the Boltzmann statistical probabilities of finding each system at each angle for three selected E-fields (see Fig. 5). Just below the boiling point of nitrogen (77.36 K), the Boltzmann populations for 77K in Figures $5 \mathrm{a}, 5 \mathrm{c}$ and $5 \mathrm{e}$ have peaks corresponding to ranges of $\theta$ with the lowest values of $E_{\text {rel }}$ in Figures 2 and 4. For an applied field of $3.6 \times 10^{7} \mathrm{~V} / \mathrm{cm}$, where in all cases only one $E_{\text {tot }}$ minima remains (see Fig. 4), all systems have a correponding single peak in their Boltzmann population fraction. For this applied field strength, the di-nitrile system shows a relatively sharp $\left(\sim 5^{\circ}\right.$ full width at half maximum FWHM) and reasonably high ( $\sim 0.45$ fraction) peak centred at $30^{\circ}$, in line with the corresponding deep 
sharp $E_{\text {tot }}$ mimimum (see Fig. 4c). In comparison, the di-fluoro and di-bromo systems have peaks with slightly lower heights $\left(<0.4\right.$ fraction), slightly larger FWHM values $\left(15^{\circ}\right.$ and $10^{\circ}$ respectively) and positions $5^{\circ}$ below and $10^{\circ}$ above the di-nitrile peak, respectively. This indicates that the higher permanent dipole of the di-nitrile system leads to a greater degree of switing selectivity.

Progresively higher E-field strengths cause an increased planarity of the dipolar aryl ring and thus the peaks tend to be shifted towards $0^{\circ}$. For a field of $1.5 \times 10^{8} \mathrm{~V} / \mathrm{cm}$ this shift is most pronounced for the di-fluoro and di-nitrile systems where the peak is centered at $0^{\circ}$. For the more stericaly hindered di-bromo system the high field peak is centered around $15^{\circ}$. For a higher ambient temperature of $298 \mathrm{~K}$ there is a corresponding slight peak broadening (by 5-10 ${ }^{\circ}$ ) and lowering of peak heights ( $50 \%)$. This effect reflects the occupation of $\theta$ conformations with higher $E_{\text {rel }}$ values. Generally, however, the increase in temperature does not lead to any significant change in the overall peaks positions or number of peaks, indicating that the conformational effects of an applied E-field would be observable under ambient conditions for all systems.
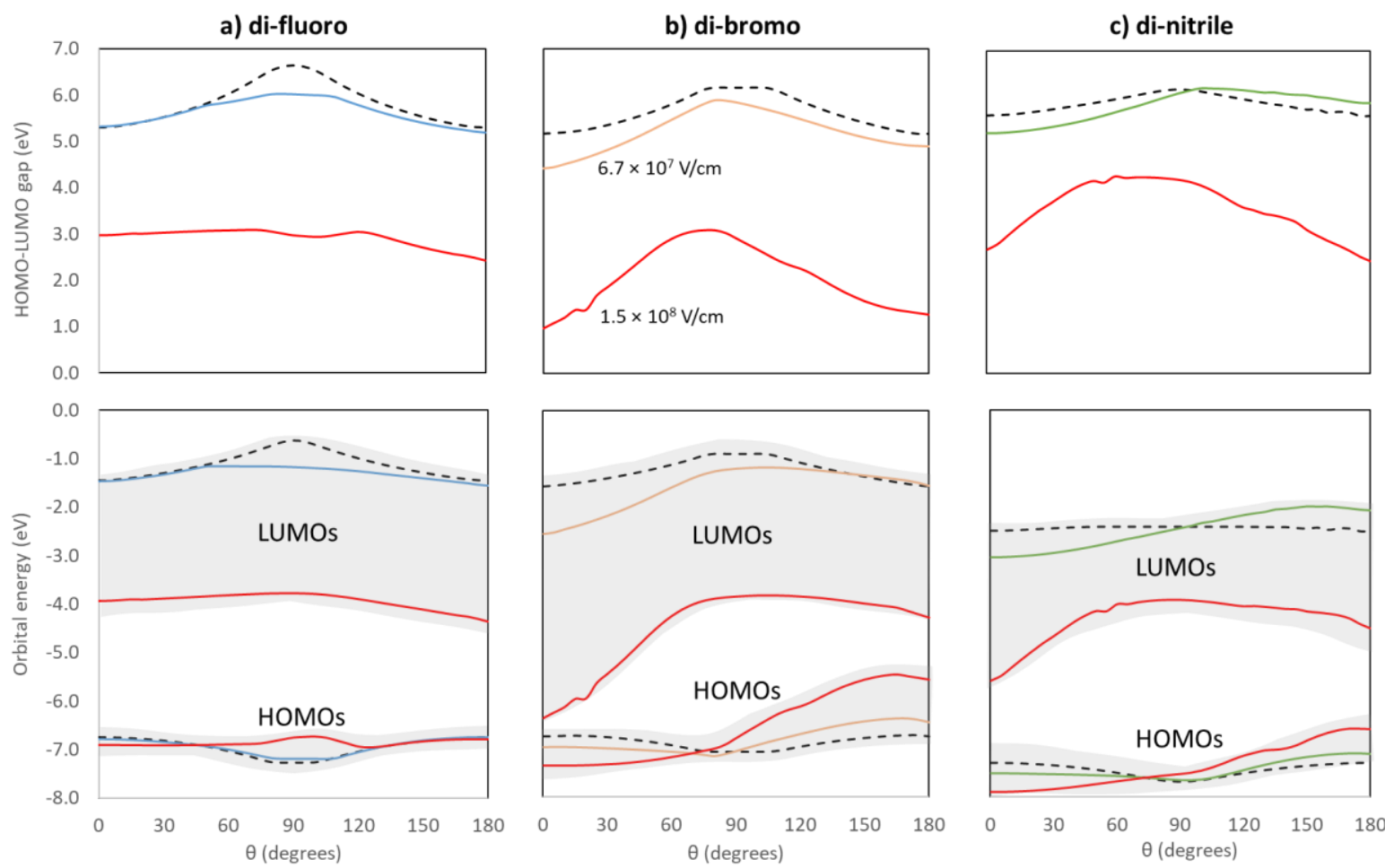

Figure 6. HOMO-LUMO gap energies (upper) and energies of individual HOMO and LUMO orbital energies with respect to $\theta$ for: a) the di-fluoro system, b) the di-bromo system, and c) the di-nitrile. All plots show data for zero field (dashed line), a field of $1.5 \times 10^{8} \mathrm{~V} / \mathrm{cm}$ (red line) and a moderate field of $6.7 \times 10^{7} \mathrm{~V} / \mathrm{cm}$ (remaining coloured line). 
The effect of both varying $\theta$ and the applied E-field strength on the electronic structure of each system is shown in Figure 6. For zero field the average gap over the full range of $\theta$ between the energy of the highest occupied molecular orbital (HOMO) and that of the lowest unoccupied molecular orbital (LUMO) is similar for the di-halogen systems ( $5.83 \mathrm{eV}$ for di-fluoro cases and $6.18 \mathrm{eV}$ for di-bromo), but is somewhat lower for the di-nitrile system $(5.01 \mathrm{eV})$. The maximum energy gap variation with respect to changes in $\theta$ is also less for the di-nitrile system $(0.5 \mathrm{eV})$ than for the di-fluro $(1.3 \mathrm{eV})$ and di-bromo $(1.0 \mathrm{eV})$ system. The most conspicuous trend in the zero field gap values with respect to $\theta$ is that all systems show a tendency to have a peak gap value close to $\theta=90^{\circ}$. This behavior is typically linked to a corresponding maximum and minimum in the $\theta$-dependent values of the respective LUMO and HOMO values. We note that $\theta=90^{\circ}$ is where $\pi$-conjugation in all systems is minimized. The destabilization of the LUMO for this highly twisted conformation strongly suggests that the LUMO tends to have a stable delocalized character through the biphenyl bridge in each system when planar. In Figure 7 we show the LUMO orbital for the case of the di-fluoro system for $\theta=0^{\circ}$ and $\theta=90^{\circ}$ to confirm this. We note that electron conduction through the biphenyl system is known experimentally to decrease with decreasing $\pi$-conjugation (i.e. $\theta$ values closer to $90^{\circ}$ ) 30 indicating that the LUMO is likely to be one of the main channels for electron conduction in these systems. The general $\theta$ dependent electronic response observed for zero field is quite robust with respect to applied E-fields and only starts to vary significantly for fields approximately $\geq 6 \times 10^{7} \mathrm{~V} / \mathrm{cm}$ (see Figure 6). This confirms that the E-field gated conformational changes realized in these systems would yield associated $\pi$ conjugation changes, and thus conductive switching, up to relatively high field strengths, without modulating the electronic structure.

For relatively high fields, the energy of the LUMO is drastically lowered for all three biphenyl systems, whereas the energy of the HOMO is less affected. As a result, a large decrease of the HOMOLUMO gap is observed for high fields. The large stabilization of the LUMOs in going from zero field to high fields originates in a drastic orbital shape change. As shown in Figure 7, the LUMO for the difluoro system for both $\theta=0^{\circ}$ and $\theta=90^{\circ}$ transforms into a Rydberg orbital under high electric fields. The virtual molecular orbitals (MOs) obtained at $\mathbf{F}_{\mathrm{x}}=1.5 \times 10^{8} \mathrm{~V} / \mathrm{cm}$ lying higher in energy than the LUMO also include a MO that is similar to the LUMO at zero field, as shown in Figure S4. The energy of this MO remains largely unchanged upon application of the electric field. The virtual MOs of the molecule at zero field, in turn, also include Rydberg states but they lie high in energy (see Figure S4). A comparison between the energies of the MOs displayed in Figure 7 with those of Figure $\mathrm{S} 4$ allows us to conclude transformation of the LUMO results from a large stabilization of one of the Rydberg orbitals upon application of large E-fields. Such E-field-induced stabilization of Rydberg orbitals is in line with the results recently found for ethylene in intense laser fields..$^{54}$ In broader terms, the strong 
decrease of the HOMO-LUMO gap found for biphenyl derivatives under large fields is also in line with the results reported in Ref. 55 for diatomic molecules, where a large decrease in the gap was observed for E-fields larger than $5 \times 10^{7} \mathrm{~V} / \mathrm{cm}$.

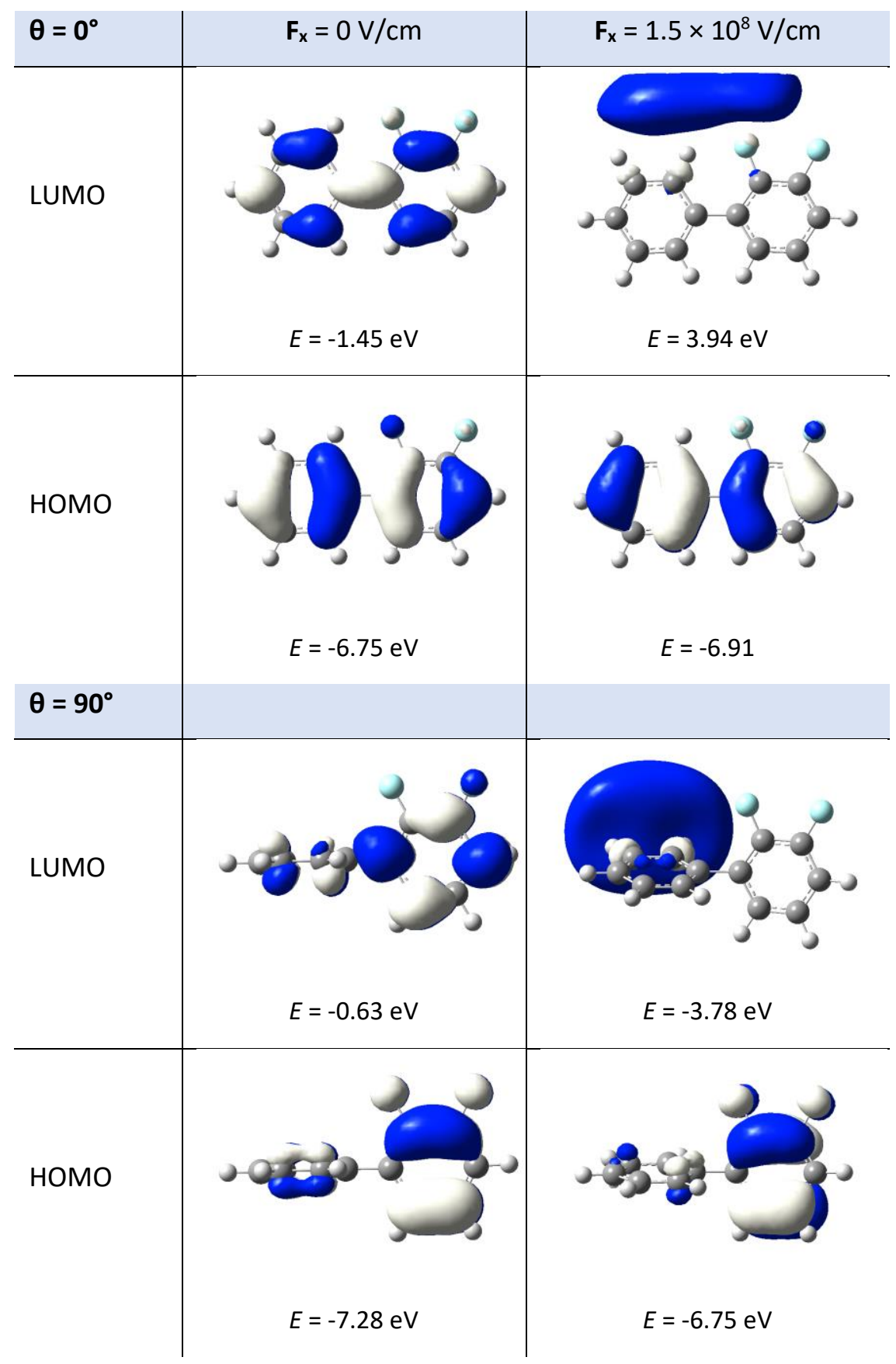

Figure 7. HOMO and LUMO orbitals and energies of the di-fluoro-biphenyl molecule for $\theta=0^{\circ}$ and $\theta=$ $90^{\circ}$, as obtained at zero field and at the highest E-field employed in this study.

The E-field induced reshuffling of MOs and the concomitant change of the energy gap demonstrates that in the high-field regime, the physical properties of biphenyl systems will be governed both by the 
induced conformation and the E-field induced changes in the electronic structure. The presence of Efield-stabilised Rydberg orbitals will likely cause significant changes in both the conduction and optical properties of biphenyl moieties.

\section{Conclusions}

We model the E-field response of the biphenyl system with three choices of dipolar substituents of one of the two aryl rings. In all cases, the E-field strengths required to induce conformational rearrangements in the twistable functionalized ring are similar to the largest E-field strengths used in silicon-based MOSFETS, and are well within those commonly found in biomolecular systems. At smaller E-field strengths the main variation in the total energy is due to the interaction of the permanent dipole with the applied field. However, the proportion of the energy change associated with the induced dipole and higher order polarization effects is always significant and rapidly becomes the dominant energy term with increasing E-field strength. This result clearly shows that E-field induced effects cannot be neglected when attempting to understand and model E-field responsive molecular switching. In line with this finding, we find that substituents that confer both a large permanent dipole moment and a modest polarizability (e.g. CN) entail that smaller E-fields are needed to trigger barrierless conformational rearrangements. For such systems the conformational switching selectivity is also likely to be enhanced. Practically, this suggests that such substituents could help design more energy efficient E-field responsive molecular switches. Looking further to eventual deployment of such switches as components in real devices we have also established that: i) the switching capability of dipolar aryl rings mediated by E-fields is robust at room temperature, ii) the electronic structure of these biphenyl systems is little affected at moderate fields - but can be strongly affected with high E-fields (which can energetically stabilise Rydberg LUMO orbitals that can drastically modify the physical properties of the system), iii) the approximate switching cycle times of twistable dipolar aryl rings estimated to be two orders of magnitude faster that the time period for the fastest commercial CPUs. These features suggest that dipolar conformational molecular switches based on substituted biphenyls or similar molecular systems could be a promising future basis for stable high performance computing in real world devices.

\section{Acknowledgments}

This work was supported by the following research grants: MICIUN/FEDER RTI2018-095460-B-I00 and CTQ2017-87773-P/AEI/FEDER (Spanish government, MINECO), MDM-2017-0767 (“María de Maeztu” program for Spanish Structures of Excellence), 2017SGR13 and 2017SGR348 (Generalitat de 
Catalunya, DURSI). R.S. acknowledges funding from MINECO under grant agreement CTQ2017-87773P/AEI/FEDER

\title{
References
}

\author{
1 J.L. Zhang, J.Q. Zhong, J. D. Lin, W.P. Hu, K. Wu, G.Q. Xu, A.T.S. Wee and W. Chen. "Towards single molecule \\ switches", Chem. Soc. Rev., 2015, 44, 2998 - 3022. \\ ${ }^{2}$ G. Ke, C. Duan, F. Huang, X. Guo. "Electrical and spin switches in single-molecule junctions", InfoMat. 2020, 2, \\ 92-112. \\ ${ }^{3}$ N. Xin, J. Guan, C. Zhou, X. Chen, C. Gu, Y. Li, M.A. Ratner, A. Nitzan, J.F. Stoddart, X. Guo, "Concepts in the \\ design and engineering of single-molecule electronic devices", Nat. Rev. Phys., 2019, 1, 211 - 230. \\ 4 J.D. Harris, M.J. Moran, I. Aprahamian, "New molecular switch architectures", Proc. Natl. Acad. Sci. USA., \\ 2018, 115, 9414-9422. \\ ${ }^{5}$ M. Baghernejad, X. Zhao, K.B. Ørns $\varnothing$, M. Füeg, P. Moreno-García, A.V. Rudnev, V. Kaliginedi, S. Vesztergom, C. \\ Huang, W. Hong, P. Broekmann, T. Wandlowski, K. S. Thygesen, M.R. Bryce, "Electrochemical Control of Single- \\ Molecule Conductance by Fermi- Level Tuning and Conjugation Switching", J. Am. Chem. Soc., 2014, 136, \\ 17922-17925.
}

${ }^{6}$ F. Schwarz, G. Kastlunger, F. Lissel, C. Egler-Lucas, S.N. Semenov, K. Venkatesan, H. Berke, R. Stadler, E. Lörtscher, "Field-induced conductance switching by charge-state alternation in organometallic single-molecule junctions", Nat. Nanotechnol., 2015, 11, 170 - 176.

${ }^{7}$ Y. Han, C. Nickle, Z. Zhang, H. P. A. G. Astier, T. J. Duffin, D. Qi, Z. Wang, E. del Barco, D. Thompson, C. A. Nijhuis, "Electric-field-driven dual-functional molecular switches in tunnel junctions", Nat. Mater., 2020, 19, $843-848$.

${ }^{8}$ E. Lörtscher, J.W. Ciszek, J. Tour, H. Riel, "Reversible and Controllable Switching of a Single-Molecule Junction", Small, 2006, 2, $973-977$.

${ }^{9}$ V. Meded, A. Bagrets, A. Arnold, F. Evers, "Molecular Switch Controlled by Pulsed Bias Voltages", Small, 2009, 5, $2218-2223$

${ }^{10}$ I. José Olavarría-Contreras, A. Etcheverry-Berríos, W. Qian, C. Gutiérrez-Cerón, A. Campos-Olguín, E.C. Sañudo, D. Dulic', E. Ruiz, N. Aliaga-Alcalde, M. Soler, H.S.J. van der Zant, "Electric-field induced bistability in single-molecule conductance measurements for boron coordinated curcuminoid compounds", Chem. Sci., $2018,9,6988-6996$.

${ }^{11}$ H. Bi, C-A. Palma, Y. Gong, P. Hasch, M. Elbing, M. Mayor, J. Reichert, J. V. Barth, "Voltage-Driven Conformational Switching with Distinct Raman Signature in a Single-Molecule Junction", J. Am. Chem. Soc., $2018,140,4835-4840$.

12 L. Meng, N. Xin, C.Hu, J. Wang, B. Gui, J. Shi, C. Wang, C. Shen, G. Zhang, H. Guo, S. Meng, X. Guo, "Sidegroup chemical gating via reversible optical and electric control in a single molecule transistor", Nat. Commun. 2019, 10, 1450. 
${ }^{13}$ Y. Zhang, P. Su, Y. Mu, G. Zhang, Y. Luo, J. Jiang, W. Hu, "Mechanism Study of Molecular Deformation of 2,2',5',2"- Tetramethylated p-Terphenyl-4,4"-dithiol Trapped in Gold Junctions", J. Phys. Chem. Lett., 2020, 11, $4456-4461$

${ }^{14}$ L. Gerhard, K. Edelmann, J. Homberg, M. Valásek, S.G. Bahoosh, M. Lukas, F, Pauly, M. Mayor, W. Wulfhekel, "An electrically actuated molecular toggle switch", Nat. Commun. 2017, 8, 14672.

${ }^{15}$ C. Tang, J. Zheng, Y. Ye, J. Liu, L. Chen, Z. Yan, Z. Chen, L. Chen, X. Huang, J. Bai, Z. Chen, J. Shi, H. Xia, W. Hong, "Electric-Field-Induced Connectivity Switching in Single-Molecule Junctions", iScience, 2020, 23, 100770.

${ }^{16}$ T. Akutagawa, H. Koshinaka, D. Sato, S. Takeda, S-I. Noro, H. Takahashi, R. Kumai, Y. Tokura, T. Nakamura, "Ferroelectricity and polarity control in solid-state flip-flop supramolecular rotators", Nat. Mater., 2009, 8, 342-347.

${ }^{17}$ Y. Zang, Q Zou, T. Fu, F. Ng, B. Fowler, J. Yang, H. Li, M. L. Steigerwald, C. Nuckolls, L. Venkataraman "Directing isomerization reactions of cumulenes with E-fields", Nat. Commun., 2019, 10, 4482.

${ }^{18}$ S. Shin, Y. Park, H. Kang, H. Kang, "E-field Effect on Condensed-Phase Molecular Systems. IV. Conformational Change of 1,2-Dichloroethane in a Frozen Molecular Solid", J. Phys. Chem. C, 2017, 121, 25342-25346.

${ }^{19}$ X. H. Qiu, G.V. Nazin, and W. Ho, "Mechanisms of Reversible Conformational Transitions in a Single Molecule", Phys. Rev. Lett., 2004, 93, 196806.

${ }^{20}$ M. Alemani, M.V. Peters, S. Hecht, K-H. Rieder, F. Moresco, L. Grill, " E-field-Induced Isomerization of Azobenzene by STM", J. Am. Chem. Soc. 2006, 128, 14446-14447.

${ }^{21}$ Y. Zhang, H. Kersell, R. Stefak, J. Echeverria, V. Iancu, U. G. E. Perera, Y. Li, A. Deshpande, K.-F. Braun, C. Joachim, G. Rapenne, S.-W. Hla, "Simultaneous and coordinated rotational switching of all molecular rotors in a network", Nat. Nanotechnol., 2016, 11, 706-713.

${ }^{22}$ G.J. Simpson, V. García-López, A. D. Boese, J. M. Tour, L. Grill, "How to control single-molecule rotation", Nat. Commun., 2019, 10, 4631.

${ }^{23}$ M. Bouatou, C. Chacon, F. Joucken, Y. Girard, V. Repain, A. Bellec, S. Rousset, R. Sporken, C. González, Y.J. Dappe, J. Lagoute, "Control of Dipolar Switches on Graphene by a Local E-field", J. Phys. Chem. C 2020, 124, 15639-15645.

${ }^{24}$ S. Mangel, M. Skripnik, K. Polyudov, C. Dette, T. Wollandt, P. Punke, D. Li, R. Urcuyo, F. Pauly, S. J. Jung, Klaus Kern, "Electric-field control of single-molecule tautomerization", Phys.Chem.Chem.Phys., 2020, 22, 6370-6375.

${ }^{25}$ A. Jaros, E. F. Bonab, M. Straka, C. Foroutan-Nejad, "Fullerene-Based Switching Molecular Diodes Controlled by Oriented External E-fields", J. Am. Chem. Soc., 2019, 141, 19644-19654.

${ }^{26}$ L. Zhang, E. Laborda, N. Darwish, B.B. Noble, J.H. Tyrell, S. Pluczyk, A.P. Le Brun, G.G. Wallace, J. Gonzalez, M.L. Coote, Simone Ciampi, "Electrochemical and Electrostatic Cleavage of Alkoxyamines", J. Am. Chem. Soc. 2018, 140, 766-774.

${ }^{27}$ A.C. Aragonès, N. L. Haworth, N. Darwish, S. Ciampi, N.J. Bloomfield, G.G. Wallace, I. Diez-Perez, M. L. Coote, "Electrostatic catalysis of a Diels-Alder reaction", Nature, 2016, 531, 88-91.

${ }^{28}$ S. Shaik, R. Ramanan, D. Danovich, D. Mandal, "Structure and reactivity/selectivity control by orientedexternal E-fields", Chem. Soc. Rev., 2018, 47, 5125-5145.

${ }^{29}$ S. Shaik, D. Danovich, J. Joy, Z. Wang, T. Stuyver, "Electric-Field Mediated Chemistry: Uncovering and Exploiting the Potential of (Oriented) E-fields to Exert Chemical Catalysis and Reaction Control", J. Am. Chem. Soc., 2020, 142, 12551-12562. 
${ }^{30}$ L. Venkataraman, J.E. Klare, C. Nuckolls, M.S. Hybertsen, M. L. Steigerwald, "Dependence of single-molecule junction conductance on molecular conformation", Nature, 2006, 442, 904-907.

${ }^{31}$ F. Pauly, J. K. Viljas, J. C. Cuevas, and Gerd Schön, "Density-functional study of tilt-angle and temperaturedependent conductance in biphenyl dithiol single-molecule junctions", Phys. Rev. B: Condens. Matter Mater. Phys., 2008, 77, 155312

${ }^{32}$ N. Xin, J. Wang, C. Jia, Z. Liu, X. Zhang, C. Yu, M. Li, S. Wang, Y. Gong, H. Sun, G. Zhang, Z. Liu, G. Zhang, J. Liao, D. Zhang, X. Guo, "Stereoelectronic Effect-Induced Conductance Switching in Aromatic Chain SingleMolecule Junctions", Nano Lett., 2017, 17, 856-861.

33 J. Valdiviezo, J.L. Palma, "Molecular Rectification Enhancement Based On Conformational and Chemical Modifications", J. Phys. Chem. C., 2018, 122, 2053-2063.

${ }^{34}$ Y-Y. Noh, R. Azumi, M. Goto, B-J. Jung, E. Lim, H-K. Shim, Y. Yoshida, K. Yase, and D-Y. Kim. "Organic Field Effect Transistors Based on Biphenyl, Fluorene End-Capped Fused Bithiophene Oligomers". Chem. Mater. 2005, 17, 3861-3870.

${ }^{35}$ S.-F. Lim, R. H. Friend, I. D. Rees, J. Li, Y. Ma, K. Robinson, A. B. Holmes, E. Hennebicq, D. Beljonne, F. Cacialli. "Suppression of Green Emission in a New Class of Blue - Emitting Polyfluorene Copolymers with Twisted Biphenyl Moieties". Adv. Funct. Mater., 2005, 15, 981-988.

${ }^{36}$ W. Li, A. Furlan, W. C. Roelofs, K. H. Hendriks, G. W. P. van Pruissen, M. M. Wienka, R. A. J. Janssen. " Wide band gap diketopyrrolopyrrole-based conjugated polymers incorporating biphenyl units applied in polymer solar cells", Chem. Commun., 2014, 50, 679-681.

${ }^{37}$ N. Cai, J. Zhang, M. Xu, M. Zhang, P. Wang. " Improving the Photovoltage of Dithienopyrrole Dye - Sensitized Solar Cells via Attaching the Bulky Bis(octyloxy)biphenyl Moiety to the Conjugated $\pi$-Linker". Adv. Funct.

Mater., 2013, 23, 3539-3547.

${ }^{38}$ Y. Su, X. Wang, L. Wang, Z. Zhang, X. Wang, Y. Song, P.P. Power, "Thermally controlling the singlet-triplet energy gap of a diradical in the solid state", Chem. Sci., 2016, 7, 6514-6518.

${ }^{39}$ D. Miguel, I.R. Márquez, L. Álvarez de Cienfuegos, N. Fuentes, S. Rodríguez-Bolivar, D.J. Cárdenas, A.J. Mota, F. Gómez-Campos, J. M. Cuerva, "Two-dimensional carbon-based conductive materials with dynamically controlled asymmetric Dirac cones", Phys. Chem. Chem. Phys., 2015, 17, 31902-31910.

${ }^{40}$ A. Troisi, M.A. Ratner, "Conformational Molecular Rectifiers", Nano Lett., 2004, 4, 591-595.

${ }^{41}$ V. Pohl, J.C. Tremblay, "Field-Induced Conformational Change in a Single-Molecule- Graphene-Nanoribbon Junction: Effect of Vibrational Energy Redistribution", J. Phys. Chem. C, 2016, 120, 28808-28819.

42 (a) P. C. Hariharan and J. A. Pople, Theor. Chim. Acta, 1973, 28, 213; (b) M. M. Francl, W. J. Petro, W. J. Hehre, J. S. Binkley, M. S. Gordon, D. J. DeFrees and J. A. Pople, J. Chem. Phys., 1982, 77, 3654.

${ }^{43} \mathrm{C}$. Adamo and V. Barone, "Toward reliable density functional methods without adjustable parameters: The PBE0 model", J. Chem. Phys., 1999, 110, 6158.

${ }^{44}$ Gaussian 09, Revision A.02, M. J. Frisch et al. Gaussian, Inc., Wallingford CT, 2016.

${ }^{45}$ T. Ytterdal, Y. Cheng, T. A. Fjeldly, Device Modeling for Analog and RF CMOS Circuit Design, John Wiley \& Sons, 2003.

${ }^{46}$ S.D. Fried, S. Bagchi, S.G. Boxer, "Extreme electric fields power catalysis in the active site of ketosteroid isomerase", Science, 2014, 346, $1510-1514$. 
47 S.D. Fried, S.G. Boxer, "Electric Fields and Enzyme Catalysis", Annu. Rev. Biochem., 2017, 86, $387-415$

${ }^{48}$ M.W. Shi, S.P. Thomas, V. R. Hathwar, A. J. Edwards, R. O. Piltz, D. Jayatilaka, G.A. Koutsantonis, J. Overgaard, E. Nishibori, B. B. Iversen, M. A. Spackman, "Measurement of Electric Fields Experienced by Urea Guest Molecules in the 18-Crown-6/Urea (1:5) Host-Guest Complex: An Experimental Reference Point for Electric-Field-Assisted Catalysis", J. Am. Chem. Soc., 2019, 141, 9, 3965-3976.

${ }^{49}$ Atoms, Molecules and Clusters in E-fields: Theoretical Approaches to the Calculation of Electric Polarizability, G. Maroulis, 2006. London: World Scientific.

${ }^{50} \mathrm{~F}$. Jensen, Introduction to Computational Chemistry, John Wiley \& Sons Ltd, 2007.

${ }^{51}$ S.M. Gutiérrez-Sanfeliciano, J.M. Schaus, "Rapid assessment of conformational preferences in biaryl and aryl carbonyl fragments", PLoS ONE, 2018, 13, e0192974.

52 E. J. Mattioli, A. Bottoni, F. Zerbetto, M. Calvaresi, "Oriented External E-fields Affect Rate and Stereoselectivity of Electrocyclic Reactions", J. Phys. Chem. C, 2019, 123, 26370-26378.

\section{${ }^{53}$ https://www.cpubenchmark.net/}

${ }^{54}$ B. Jochim, R. Siemering, M. Zohrabi, O. Voznyuk, J. B. Mahowald, D. G. Schmitz, K. J. Betsch, B. Berry, T. Severt, N.G. Kling, T. G. Burwitz, K. D. Carnes, M. F. Kling, I. Ben-Itzhak, E. Wells, R. de Vivie-Riedle, "The importance of Rydberg orbitals in dissociative ionization of small hydrocarbon molecules in intense laser fields", Sci. Rep., 2017, 7:4441.

${ }^{55}$ F. N. N. Pansini, F. A. L. de Souza, C. T. Campos, "Molecules Under External E-field: On the Changes in the Electronic Structure and Validity Limits of the Theoretical Predictions", J. Comput. Chem., 2018, 39, 1561-1567. 